\section{Reflecting the Debates of Updating the Prostate Cancer Guidelines}

The NCCN Prostate Cancer Clinical Practice Guidelines in Oncology are revised at least annually using teleconferences, and every 3 years, they are examined in greater detail in face-to-face panel meetings. The 2006 face-to-face meeting addressed 5 major issues. To give guidelines users an appreciation for the process used to make the fairly substantial changes in the 2007 Guidelines, this issue of the Journal of the National Cancer Network (JNCCN) includes point/counterpoint manuscripts for 3 of those 5 debates.

\section{Can Life Expectancy Be Estimated Accurately?}

Life expectancy is estimated using the Minnesota Metropolitan Life Insurance Tables or the Social Security Administration Life Expectancy tables. It can then be adjusted for individual patients by adding or subtracting $50 \%$ based on whether one believes the patient is in the healthiest quartile or the unhealthiest quartile, respectively. ${ }^{1}$ Thus, treatment recommendations can change if a man was judged to be in poor or excellent health.

The perceived need to incorporate life expectancy estimation resulted in the panel's first point/counterpoint debate, the flavor of which was captured in the articles in this issue by Clayton and Urban and McCloskey and Kuettel. The debate resulted in the panel reaching several conclusions, and the 2007 guidelines, for the first time, included a "Principles of Life Expectancy Estimation" table (see page 659).

- Prediction of life expectancy in individuals (not groups) is not possible.

- The Panel was concerned that patients would receive information about their life expectancy and not have the ability to consider it appropriately.

- The Panel agreed that accurate life expectancy estimation is highly desirable.

- The NCCN Senior Adult Oncology Guidelines use the Social Security Administration life expectancy table with life expectancy adjustment by quartile of health determined numerically using the VES13 instrument. The Panel thought it unclear how that instrument is used to determine quartiles of health and found its use impractical in the clinical setting.

- The Panel felt it best to allow the quartile assessment to remain the gestalt of the clinician.

\section{Active Surveillance or Immediate Active Treatment?}

Increasing evidence suggests that more patients are being treated for clinically localized prostate cancer than will die of the disease. A growing literature attempts to define "clinically insignificant prostate cancer," and consensus seems that clinically insignificant cancer has Gleason score less than 8 , less than $50 \%$ of the total number of, or less than 3 prostate biopsies positive, less than $50 \%$ of any biopsy positive, and PSA less than 10 or less than $15 .^{2-6}$ Using similar guidelines, most patients remained on expectant management after 2 years, and approximately half remained on expectant management after 4 years. Most patients converted from expectant management to active treatment for reasons other than prostate cancer progression. ${ }^{6}$ These findings generated the second debate, discussed in the articles by Klotz and Leob and Catalona.

The discussion resulted in modification of "Principles of Expectant Management" (see page 664).

- The risks of watchful waiting include chance of missed opportunity for cure; nervesparing may be more difficult which may reduce the chance of potency preservation; and anxiety.

- The benefits of watchful waiting include avoidance of possible treatment-related side effects, which may result from a treatment that was unnecessary.

- The Panel agreed that we are over-treating men with prostate cancer, especially older men with favorable prostate cancer.

- The Panel remains uncertain whether PSA doubling time or PSA velocity provides a more accurate indicator of tumor group rates.

- Drs. Klotz and Catalona contrasted the conversion rate from watchful waiting to treatment, which is approximately $10 \%$ in Canada and 35\% to $50 \%$ in the United States.

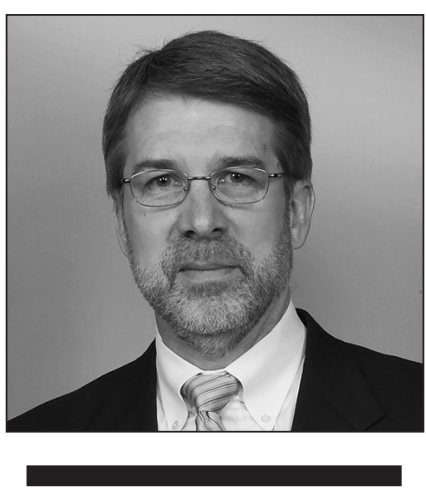

James L. Mohler, MD

James L. Mohler, MD, is Chair of the Department of Urologic Oncology, Leader of the Prostate Program, and Professor of Oncology at Roswell Park Cancer Institute. Dr. Mohler also serves as Professor of Urology at the University at Buffalo (UB) School of Medicine and Biomedical Sciences. Dr. Mohler maintains a clinical practice, focusing on prostate cancer and robotassisted laparoscopic surgery. In addition to serving as chair of the NCCN Prostate Cancer Panel, he has authored more than 150 publications and book chapters, serves on the editorial board of The Prostate and The Journal of Robotic Surgery, and is a reviewer for numerous cancer journals.

The ideas and viewpoints expressed in this editorial are those of the author and do not necessarily represent any policy, position, or program of the NCCN. 
- The Panel felt that when younger and healthier men choose watchful waiting, they should be followed more actively, with PSAs measured as often as 3 times monthly and prostate biopsies as often as annually, whereas older and less healthy men can be followed less frequently.

\section{Open Radical Retropubic Prostatectomy Versus Robotic and Laparoscopic}

Radical retropubic prostatectomy is the gold standard treatment for clinically localized prostate cancer. Laparoscopic and robot-assisted techniques have brought minimally invasive surgery to urologic oncology, with the first robotic prostatectomy performed in the United States at Henry Ford Hospital in November 2000. In fact, estimates are that less than $1 \%$ of radical prostatectomies were performed robotically in $2002,4 \% 2003,15 \%$ in $2004,24 \%$ in 2005 , and $37 \%$ in 2006. The panel accepts the rapid growth of robot-assisted radical prostatectomy but desires careful evaluation of its use. Early reports of outcome data suggest that robot-assisted radical prostatectomy hastens recovery of activities of daily living, urinary control, and erectile function. ${ }^{7,8}$

A spirited debate, framed by the point/counterpoint described in the articles by Ellis and Lange and Kawachi, allowed the panel to revise the 2007 guidelines to "laparoscopic and robotic radical prostatectomy are under development. In highly experienced hands the results may be comparable to or better than open surgical approaches."

\section{Other Debates}

Finally, the Panel conducted 2 additional debates. The first was whether salvage radiotherapy should be offered to more men with detectable PSA after radical prostatectomy or whether the side effects and poor utility of salvage radiation therapy do not warrant a liberalization of the use of salvage radiotherapy. The second was whether men with intermediate risk prostate cancer should receive ADT and dose escalation or whether ADT and dose escalation are unnecessary in intermediate risk prostate cancer.

These presentations and the discussion that resulted allowed revisions of the sections on radiation therapy in the 2007 guidelines. The 2008 guidelines will recommend increased use of nomograms to enhance patient education regarding controversies in treatment of clinically localized prostate cancer and for counseling men regarding salvage radiation therapy for postprostatectomy biochemical progression. Careful consideration of new information as it becomes available and spirited discussion of differences in opinions among experts at member institutions should allow the NCCN treatment guidelines in general, and the prostate cancer guidelines specifically, to inform clinicians and their patients and facilitate intelligent treatment decisions while remaining current through at least annual updates.

\section{References}

1. Howard D H. Life expectancy and the value of early detection. J Health Econ 2005;24:891.

2. Epstein JI, Walsh PC, Carmichael M, et al. Pathologic and clinical findings to predict tumor extent of nonpalpable (stage T1c) prostate cancer. JAMA 1994;271:368.

3. Epstein JI, Chan DW, Sokoll LJ, et al. Nonpalpable stage T1c prostate cancer: prediction of insignificant disease using free/total prostate specific antigen levels and needle biopsy findings. J Urol 1998;160:2407.

4. Cheng L, Jones TD, Pan CX, et al. Anatomic distribution and pathologic characterization of small-volume prostate cancer $(<0.5 \mathrm{ml})$ in whole-mount prostatectomy specimens. Mod Pathol 2005;18:1022.

5. Cheng L, Poulos CK, Pan CX, et al. Preoperative prediction of small volume cancer (less than $0.5 \mathrm{ml}$ ) in radical prostatectomy specimens. J Urol 2005;174:898.

6. Choo R, Klotz L, Danjoux C, et al: Feasibility study: watchful waiting for localized low to intermediate grade prostate carcinoma with selective delayed intervention based on prostate specific antigen, histological and/or clinical progression. J Urol 2002;167:1664.

7. Menon M, Hemal AK. Vattikuti Institute prostatectomy: a technique of robotic radical prostatectomy: experience in more than 1000 cases. J Endourol 2004;18:611.

8. Ahlering TE. Robotic prostatectomy: is it the future? Urol Oncol 2006;24:1. 